\title{
Análisis espacio-temporal de las áreas verdes urbanas de la Zona Metropolitana de Monterrey, México
}

\section{Space-time analysis of urban green areas in the Monterrey Metropolitan Zone, Mexico}

\begin{tabular}{|c|}
\hline $\begin{array}{l}\text { Ulises } \\
\text { Quiñones } 1 * \sqrt[0]{0}, \\
\text { Gyorgy Eduardo Manzanilla- } \\
\text { Quijada2 }^{2} \text {, } \\
\text { Patricia Delgado-Valerio }\end{array}$ \\
\hline $\begin{array}{l}\text { }{ }^{1} \text { Universidad Michoacana } \\
\text { de San Nicolás de Hidalgo, } \\
\text { Facultad de Agrobiología } \\
\text { "Presidente Juárez", Avenida } \\
\text { Revolución esquina con Berlín } \\
\text { s/n, Colonia Viveros, CP. } \\
60170 \text {, Uruapan, Michoacán, } \\
\text { México. } \\
\text { 'Universidad Autónoma de } \\
\text { Nuevo León, Facultad de } \\
\text { Ciencias Forestales, Carretera } \\
\text { Nacional km 145, CP. } 67700 \text {, } \\
\text { Linares, Nuevo León, México. }\end{array}$ \\
\hline
\end{tabular}

Nota cientifica

Recibido: 08 de julio 2020

Aceptado: 15 de marzo 2021

Como citar: ManzanillaQuiñones $U$, Manzanilla-

Quijada GE, Delgado-Valerio P (2021) Análisis espaciotemporal de las áreas verdes urbanas de la Zona Metropolitana de Monterrey, México. Ecosistemas y Recursos Agropecuarios 8(1): e2676. DOI: 10.19136/era.a8n1.2676
RESUMEN. Las áreas verdes urbanas (AVU) proveen de relevantes servicios ecosistémicos a las ciudades, desafortunadamente, estas son vulnerables a los procesos de urbanización. El objetivo del estudio fue analizar bajo un enfoque espacio-temporal las AVU de la Zona Metropolitana de Monterrey (ZMM). Se utilizaron imágenes satelitales Landsat y Global Forest Change para estimar las AVU y la calidad ambiental de la ZMM para el periodo 2000-2019. Se encontró una tendencia $(p<0.01)$ de reducción de AVU de 2001 a 2019. La calidad ambiental estimada paso de 13.21 (2000) a $7.75 \mathrm{~m}^{2}$ (2019). La superficie de AVU se perdió a un ritmo de $0.29 \mathrm{~m}^{2}$ por habitante por año. Los municipios de Juárez, Monterrey, Apodaca y Guadalupe fueron los que mayor AVU perdieron. La conservación y protección de AVU deben ser actividades prioritarias para las autoridades civiles de los municipios de la ZMM, específicamente, en el municipio de Juárez.

Palabras clave: Áreas urbanas, calidad ambiental, geografía urbana, sistemas de información geográfica, vegetación urbana.

ABSTRACT. Urban green areas (UGA) provide relevant ecosystem services to cities, unfortunately, they are vulnerable to urbanization processes. The objective of this study was to analyze under a spatio-temporal approach the UGA of the Metropolitan Zone of Monterrey (MZM). Landsat and Global Forest Change satellite images were used to estimate the UGA and environmental quality of the MZM for the period 2000-2019. A trend $(p<0.01)$ of UGA reduction was found from 2001 to 2019 . The estimated environmental quality decreased from 13.21 (2000) to $7.75 \mathrm{~m}^{2}$ (2019). The UGA area was lost at a rate of $0.29 \mathrm{~m}^{2}$ per inhabitant per year. The municipalities of Juarez, Monterrey, Apodaca and Guadalupe were the ones that lost UGA. The conservation and protection of UGA should be priority activities for the civil authorities of the municipalities of the MZM, specifically, in the municipality of Juarez.

Key words: Urban areas, environmental quality, urban geography, geographic information systems, urban vegetation. 


\section{INTRODUCCIÓN}

En la actualidad la creciente expansión de la mancha urbana que experimentan las grandes ciudades ha provocado alta demanda en la construcción de viviendas, condominios y edificios, lo que ha propiciado problemas ambientales para los habitantes de dichas ciudades (Garza 2003, Castro 2005). Como son menor calidad del aire (mayor cantidad de gases contaminantes suspendidos en la atmósfera), reducción de los servicios ecosistémicos (captura de carbono y liberación de oxígeno), alteraciones en el clima local (cambio climático a escala local) y en general menor calidad de vida ambiental debido a la reducción de áreas verdes urbanas por los procesos de urbanización (Blancarte-Siqueiros et al. 2020, Manzanilla-Quiñones et al. 2021).

Estudios realizados sobre la pérdida de la cobertura vegetal, con técnicas de percepción remota y de sistemas de información geográfica (EspañaBoquera y Champo-Jiménez 2016) han demostrado que la vegetación presenta diferentes respuestas espectrales, las cuales varían de acuerdo con el tipo de pigmento, estructura celular, humedad o fenología de la planta (Rouse et al. 1974). Por lo general, es la clorofila la que absorbe la radiación electromagnética de las longitudes de onda del azul y rojo, pero solamente refleja la banda verde (Pérez y Muñoz 2006). A través de este tipo de estudios se ha logrado identificar tipos de vegetación (bosques, selvas, pastizales, entre otras) y su comportamiento histórico (pérdidas y/o disturbios) a través del tiempo en el paisaje de una región en particular (Hansen et al. 2013, FAO 2018). Siendo la detección de cambios en la cobertura vegetal a partir de una secuencia multitemporal de imágenes de satélite una de las aplicaciones más importantes en percepción remota y en sistemas de información geográficas (Molina et al. 2019).

Las áreas verdes urbanas (AVU) son ecosistemas que brindan servicios ecosistémicos importantes para los habitantes de las grandes urbes o metrópolis del mundo, desafortunadamente estas están experimentando rápidos procesos de urbanización, lo que ha ocasionado reducciones en su superficie y la calidad de los servicios ambien- tales que proporcionan (Priego 2011). En general, las AVU brindan beneficios ambientales como la mejora en la calidad del aire, captura de carbono, captación de agua, forestación urbana, control de inundaciones y restauración de tierras (Castro 2005). Debido a las tendencias actuales de rápido crecimiento poblacional en las grandes ciudades, la preservación y gestión de las AVU se pone en riesgo (Reyes 2012).

La relación entre las AVU y la calidad ambiental de vida de los habitantes ha sido investigada principalmente en países desarrollados de Europa y América (Haslauer et al. 2015, Kweon et al. 2016), pero, este tipo de estudios son recientes para México (Navarro-Tec et al. 2018, Blancarte-Siqueiros et al. 2020). Diversos estudios coinciden en que una mayor cobertura de AVU generan mejor calidad de vida ambiental y de salud para los habitantes (van den Berg et al. 2010, Blancarte-Siqueiros et al. 2020). Motivo por el cual, el estudio de las AVU debe ser relevante para la planeación estratégica de desarrollo urbano de las grandes ciudades como México, Guadalajara y Monterrey (González y Larralde 2019). Al respecto, la Zona Metropolitana de Monterrey (ZMM) es un área industrial de alta relevancia para la economía en México y es una de las zonas más pobladas del país (INEGI 2017, CONAPO 2018, González y Larralde 2019), dicha ZMM no cuenta con estimaciones actuales ni análisis de tendencias de sus AVU con las que cuenta. Por lo que el objetivo del presente estudio fue analizar bajo un enfoque espacio-temporal las AVU de la Zona Metropolitana de Monterrey (ZMM).

\section{MATERIALES Y MÉTODOS}

El área de estudio comprende la parte urbana ubicada en la Zona Metropolitana de Monterrey (Figura 1), conformada por los municipios de Monterrey, Santiago, Santa Catarina, Cadereyta de Jiménez, Juárez, San Pedro Garza García, García, San Nicolás de los Garza, General Escobedo, Guadalupe, Apodaca y Carmen (INEGI 2017), en los cuales se concentra una población de 4735 700 habitantes, de acuerdo con las estimaciones proyectadas para 2019 por el Consejo Nacional de 


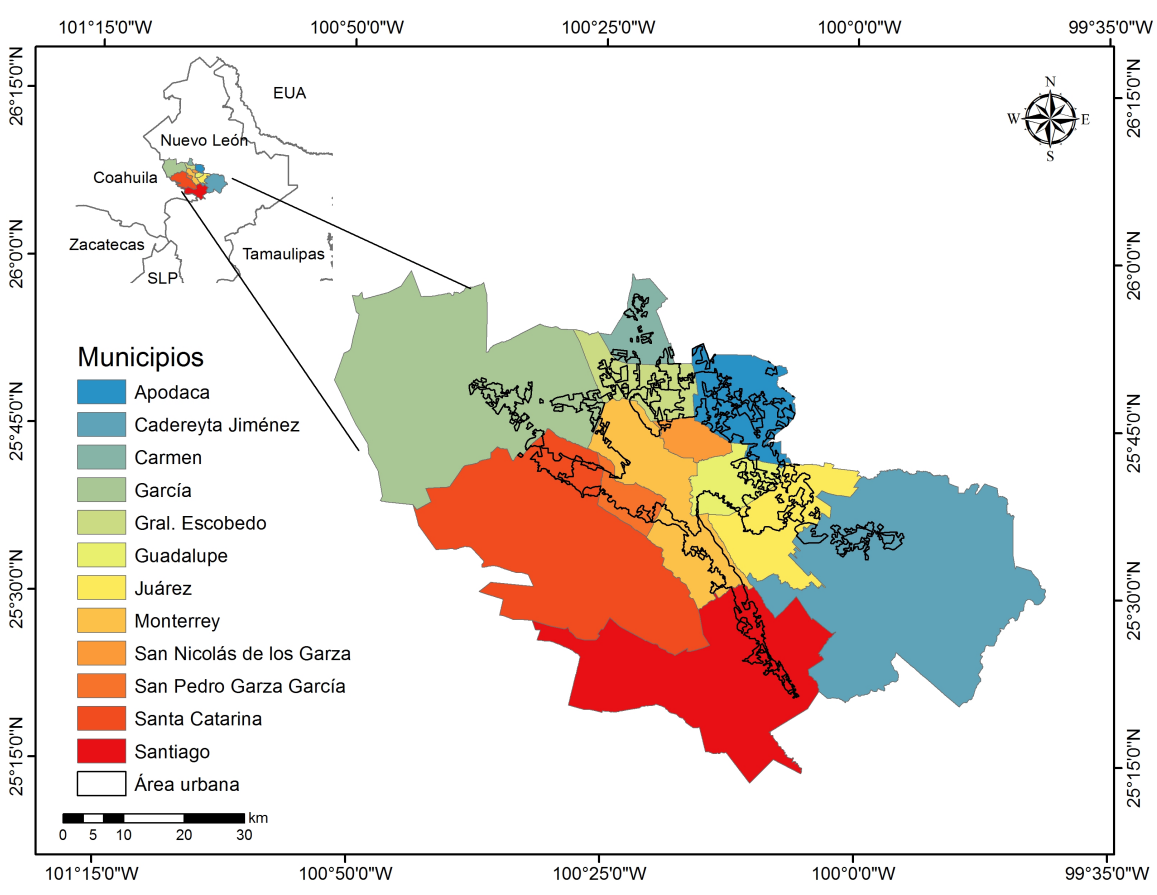

Figura 1. Ubicación geográfica del área de estudio.

Población (CONAPO 2018).

\section{Análisis espacial}

Se descargaron las bandas multiespectrales de la imagen satelital Landsat 5 de la plataforma del Servicio Geológico de los Estados Unidos (USGS) para el año 2000 (como punto de referencia para realizar las comparaciones de pérdidas en superficies de AVU) y los productos satelitales del Global Forest Change (GFC) de la plataforma Global Forest Change (Hansen et al. 2013) para el periodo de 2001 al 2019, tanto la imagen como los productos satelitales se descargaron con una resolución espacial de $30 \mathrm{~m}$ de tamaño de pixel.

\section{Procesamiento satelital}

Debido a que las bandas multiespectrales presentan errores al momento de su captura por parte del sensor del satélite, se realizaron correcciones de tipo atmosférico a las bandas roja (B3) e infrarroja cercana (B4), para eliminar los objetos oscuros y efectos derivados de la dispersión atmosférica (Bruce y Hilbert 2004). Las correcciones se realizaron con ayuda de la herramienta de semiclasificación automática de imágenes satelitales del programa QGIS versión 3.2.3 (QGIS 2018).

Con las bandas satelitales infrarroja cercana (B4) y roja (B3) se generó un índice de vegetación diferencial normalizada (IVDN) para identificar las áreas de vegetación dentro de la zona urbana de Monterrey, mediante la aplicación de la siguiente fórmula (Rouse et al. 1974):

$$
I V D N=B 4-B 3 / B 4+B 3
$$

La información del índice representa un conjunto de valores que oscilan de -1 a 1 , donde valores negativos representan cuerpos de agua, valores de 0 a 0.2 áreas desprovistas 0 con escasa vegetación, mientras que valores cercanos a 1 sugieren la presencia de zonas de densa vegetación como bosques y selvas.

Se utilizó el polígono de la ZMM para recortar el IVDN al tamaño del área de estudio. Para estimar las áreas verdes urbanas en la ZMM se aplicó un umbral de corte IVDN >0.2, la aplicación del umbral permitió descartar las áreas sin vegetación y los 
cuerpos de agua presentes en el área de estudio. Posteriormente, se transformó el polígono de estudio de formato ráster a vectorial, con el fin de estimar las superficies (ha) de áreas verdes urbanas presentes en la ZMM. Los resultados sobre la condición inicial de superficies de áreas verdes urbanas del 2000, se estimaron en valores por hectáreas (ha). Todos estos geoprocesos se realizaron con el programa QGIS versión 3.2.3 (QGIS 2018).

\section{Análisis multitemporal}

Se empleó información satelital disponible de la plataforma Global Forest Change (Hansen et al. 2013) del período 2001-2019 para estimar la pérdida en superficie de las AVU en la ZMM. Las pérdidas de AVU se calcularon por año y se realizó un análisis de tendencias del periodo 2001 al 2019 mediante la aplicación de la prueba no paramétrica de Mann-Kendall en el programa $R$ versión 4.0 (Core Team 2020) para conocer la tendencia de las superficies en hectáreas de las AVU de la ZMM.

\section{Calidad ambiental}

La relación de la calidad ambiental de los habitantes de la Zona Metropolitana de Monterrey se estimó al dividir la superficie de AVU en $\mathrm{m}^{2}$ entre la cantidad total de habitantes $(P)$ reportada por INEGI (2000) durante el 2000 y la CONAPO (2018) para 2019 en la ZMM, por medio de la siguiente fórmula:

$$
\text { Calidad ambiental }=A V U / P
$$

Dónde: $\mathrm{AVU}=$ áreas verdes urbanas y $\mathrm{P}=$ Cantidad total de habitantes para determinado año.

Se comparó la calidad ambiental de la condición inicial (2000) y final (2019), y se identificaron los años que mayor y menor reducción de superficie (ha) de AVU se presentó en la ZMM, también, se identificaron los municipios que mayor reducción presentaron en superficie de AVU durante el periodo evaluado.

\section{RESULTADOS Y DISCUSIÓN}

Se estimaron 4176.9 ha de AUV para el 2000, pero, esta cantidad se redujo hasta 3668.63 ha para el 2019 (Figura 2), siendo el 2008 (55.16 ha) y 2019 (5.09 ha) los años que mayor y menor reducción de AVU presentaron en la ZMM. Los resultados de $7.75 \mathrm{~m}^{2}$ obtenidos son inferiores a los $9 \mathrm{~m}^{2}$ indicados como valor mínimo de AVU por habitante establecido por la Organización Mundial de la Salud (OMS), aunque durante el 2000 la ZMM contaba con $13.21 \mathrm{~m}^{2}$ de $A V U$, lo que indica que dicha superficie se perdió a un ritmo constante de $0.29 \mathrm{~m}^{2}$ por habitante por cada año. Tendencias similares de reducción de AVU han sido halladas por NavarroTec et al. (2018) para el periodo 2001-2016 en la zona metropolitana de Mérida, Yucatán. Mientras que Blancarte-Siqueiros et al. (2020) encontraron que las AVU de la Ciudad de Durango fueron insuficientes $\left(3.67 \mathrm{~m}^{2}\right)$ para los habitantes de dicha ciudad, en tanto que Pena-Salmon y Rojas-Caldelas (2009), Galindo-Bianconi y Victoria-Uribe (2012) y Jiménez et al. (2013) hallaron superficies de 2.1, 5 y 6.2 $\mathrm{m}^{2}$ de AVU, respectivamente para Mexicali, Toluca y Monterrey. Lo que indica que esta reducción e insuficiencia de áreas verdes en la ZMM y ciudades de Mérida, Durango, Mexicali, Toluca y Monterrey se debe principalmente a los acelerados procesos de urbanización e industrialización experimentados en las últimas décadas en las áreas periféricas de las zonas metropolitanas de México (Garza 2003, Reyes 2012, González y Larralde 2019).

Los resultados de la prueba de Mann-Kendall indicaron una tendencia significativa $(p<0.01)$ en reducción de las AVU (Figura 3), con un promedio estimado de 26.75 ha por año, lo que equivale a una pérdida de $0.29 \mathrm{~m}^{2}$ de AVU por habitante por cada año en la ZMM. Siendo los Municipios de Juárez (139.7 ha), Monterrey (98.3 ha), Apodaca (97.1 ha) y Guadalupe (43.5 ha) los más afectados. Lo que indica que el proceso de urbanización ha tenido una mayor intensidad en estos municipios (Figura 4) siendo la zona oriente, la que mayor proceso de expansión urbana e industrial experimentó del 2001 al 2019 (Figura 5). Por lo que a futuro es probable que el crecimiento urbano e industrial continúe reduciendo la superficie de AVU en la ZMM (Secretaría de Desarrollo Sustentable del Estado de Nuevo León 2017). De acuerdo con proyecciones de crecimiento mundial 


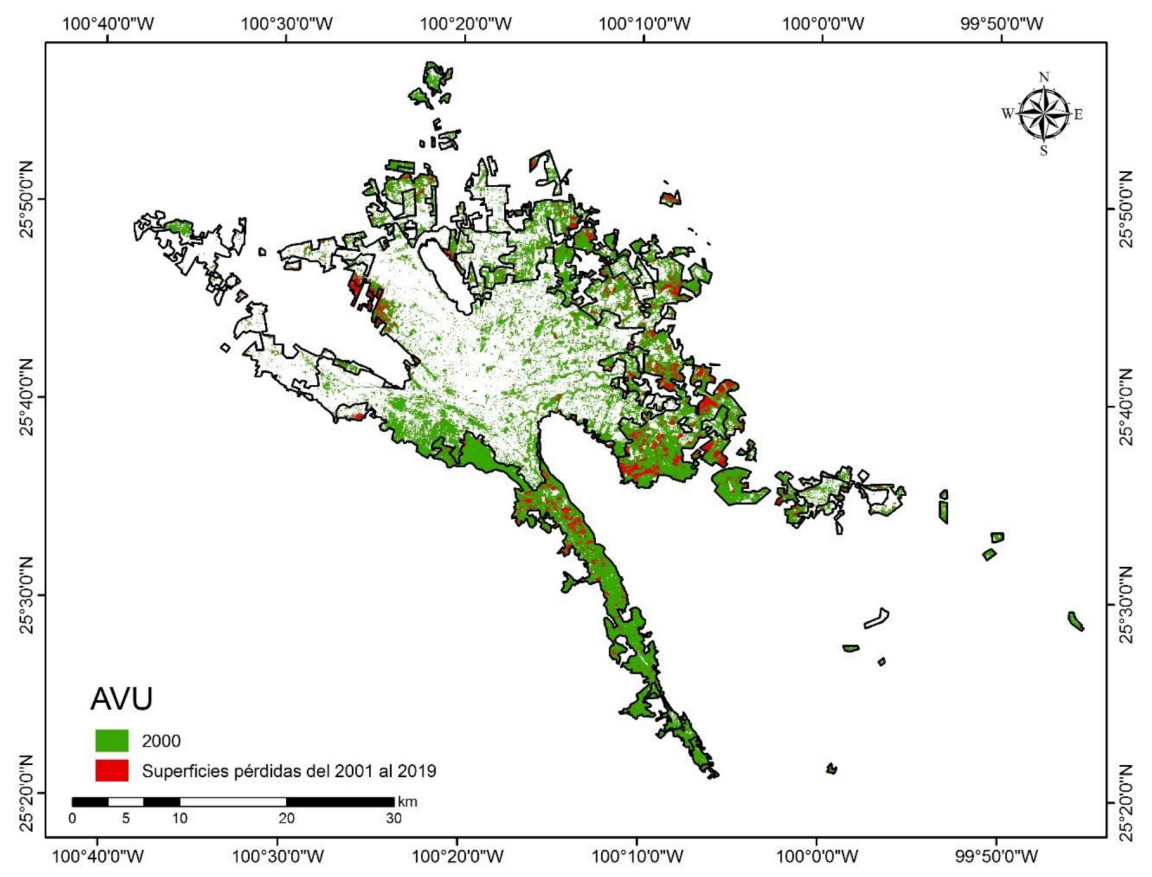

Figura 2. AVU estimadas durante el periodo de 2000 al 2019 en la ZMM.

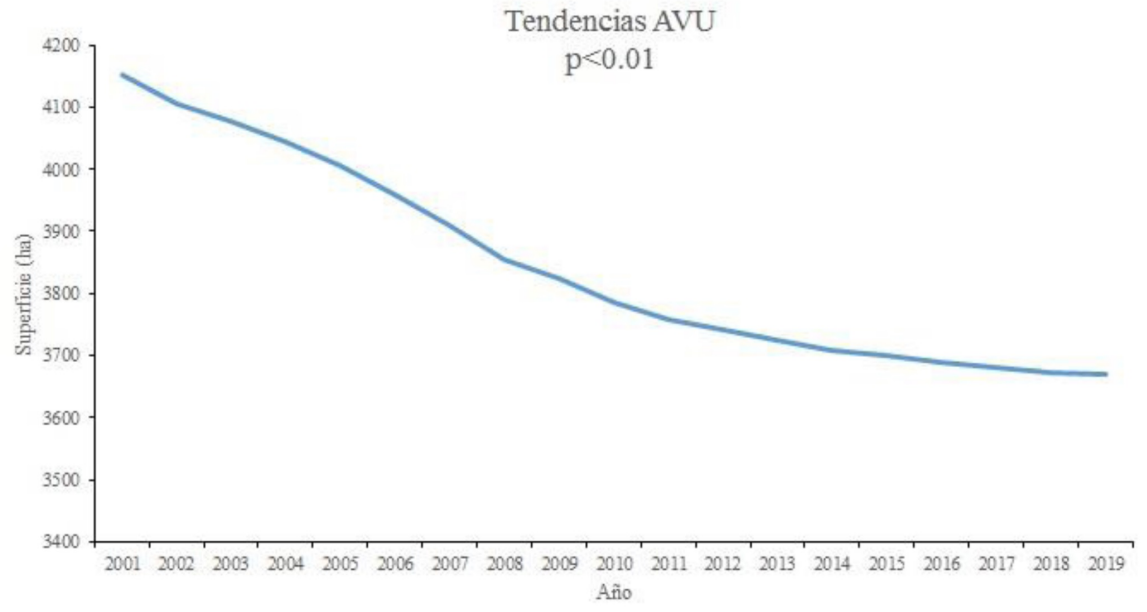

Figura 3. Análisis de tendencias de la superficie de AVU del periodo 2000 al 2019 en la ZMM.

urbano, para 2050, la proporción de personas que vivirán en las zonas urbanas será de $69.6 \%$ (Marans 2015, UN 2019). Esta constante tendencia en el crecimiento urbano traería consigo una alta demanda en la construcción de viviendas, servicios básicos y ecosistémicos, los cuales deberán ser prioridad dentro de las actividades de planeación y desarrollo urbano a futuro en las periferias de la ZMM.
Las AVU proveen muchos servicios ecosistémicos que van desde la reducción del ruido, regulación del clima, recreación y hábitats para fauna, hasta beneficios culturales y de salud (Castro 2005, van den Berg et al. 2010). Sin embargo, la calidad ambiental estimada durante el 2000-2019 paso de 13.21 a $7.75 \mathrm{~m}^{2}$ de AVU por habitante, lo que evidencia una reducción de $5.46 \mathrm{~m}^{2}$ de AVU por habitante 


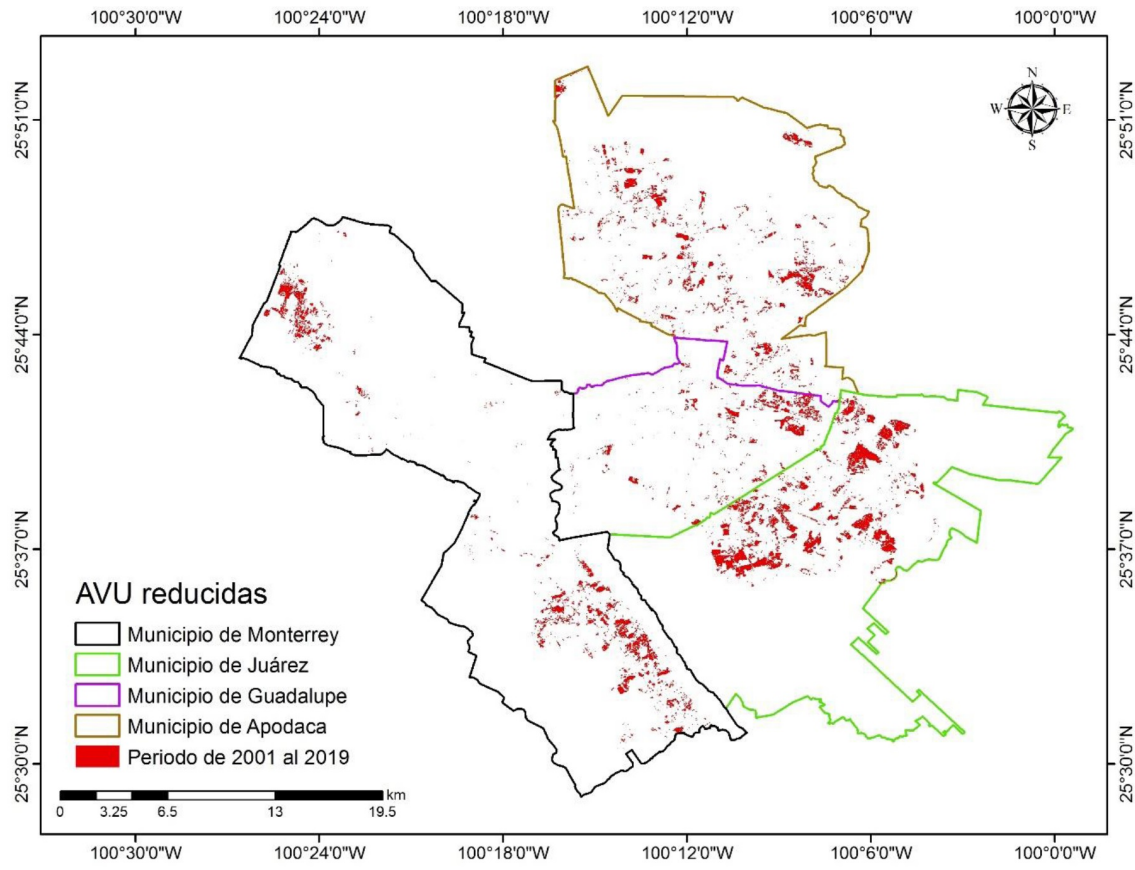

Figura 4. Superficies de AVU reducidas en los Municipios de Monterrey, Juárez, Apodaca y Guadalupe durante el periodo de 2001 al 2019.

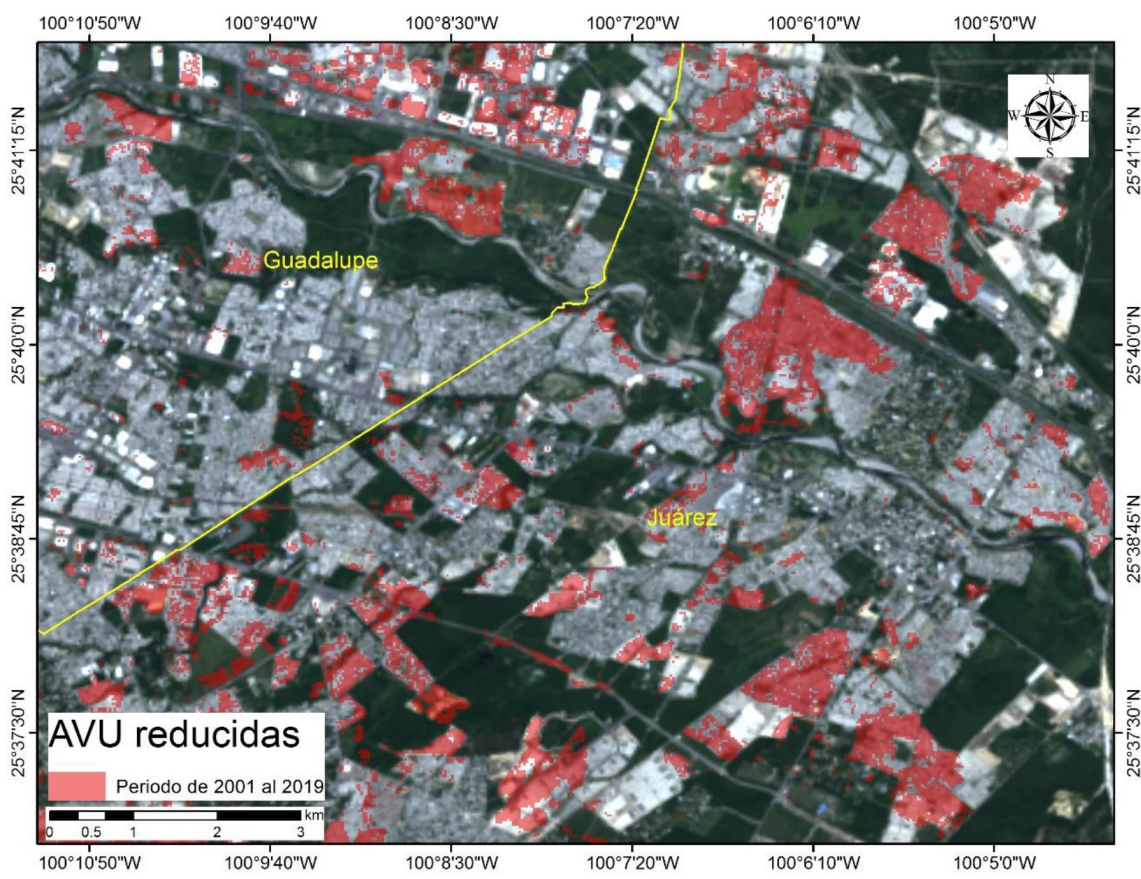

Figura 5. Superficies reducidas de AVU durante el periodo de 2001 a 2019 en la zona oriente de la ZMM. 
en la ZMM. Sí bien las superficies de AVU estimadas en este estudio son insuficientes para una óptima calidad ambiental de vida de cada habitante, los servicios ecosistémicos como la captura de carbono, liberación de oxígeno y termorregulación del clima son de gran importancia para la metrópoli (Castro 2005, Reyes 2012, Blancarte-Siqueiros et al. 2020). Por lo que las actividades de conservación y restauración ayudarían sustancialmente en mejorar la calidad ambiental de los habitantes de la ZMM.
Se encontró que los municipios de Juárez, Monterrey, Apodaca y Guadalupe fueron los sitios que mayor proceso de urbanización y pérdida de AVU presentaron durante los últimos años (2001-2019). La conservación y protección de las AVU deben ser actividades prioritarias para las autoridades civiles de los municipios de la ZMM, específicamente, en el municipio de Juárez, el cual experimentó reducciones sustanciales de AVU durante un periodo de 19 años.

\section{LITERATURA CITADA}

Blancarte-Siqueiros RH, Pérez-Verdín G, Cortes-Ortiz A (2020) La relación entre la calidad de vida, sentido de pertenencia y áreas verdes en ambientes urbanos en la ciudad de Durango, México. Revista Chapingo Serie Ciencias Forestales y del Ambiente 26: 97-111.

Bruce C, Hilbert D (2004) Pre-processing methodology for application to landsat TM/ETM+ imagery of the wet tropics. CSIRO Tropical Forest Research Centre and Rainforest CRC, Cairns. Australia. 44p.

Castro S (2005) Evaluación de un índice para valorar las áreas verdes urbanas: su aplicación y análisis en la localidad del barrio Dent y Altos del Escalante con una perspectiva geográfica. Reflexiones 84: 107-125.

CONAPO (2018) Proyecciones de la población de México y de las entidades federativas 2016-2050. Consejo Nacional de Población. https://datos.gob.mx/busca/dataset/proyecciones-de-la-poblacion-de-mexico-y-delas-entidades-federativas-2016-2050. Fecha de consulta: 15 de mayo de 2020.

España-Boquera ML, Champo-Jiménez O (2016) Proceso de deforestación en el municipio de Cherán, Michoacán, México (2006-2012). Madera y Bosques 22: 141-153.

FAO (2018) El estado de los bosques del mundo. Las vías forestales hacia el desarrollo sostenible. Food and Agriculture Organization. Roma, Italia. 132p.

Galindo-Bianconi AS, Victoria-Uribe R (2012) La vegetación como parte de la sustentabilidad urbana: beneficios, problemáticas y soluciones para el Valle de Toluca. Quivera 14: 98-108.

Garza VG (2003) La urbanización de México en el siglo XX. Primera edición. El Colegio de México. México. $208 p$.

González AS, Larralde CAH (2019) La forma urbana actual de las zonas metropolitanas en México: indicadores y dimensiones morfológicas. Estudios Demográficos y Urbanos 34: 11-42.

Hansen MC, Potapov PV, Moore R, Hancher M, Turubanova S, Tyukavina A, Thau D, Stehman SV, Goetz SJ, Loveland TR, Kommareddy A, Egorov A, Chini L, Justice CO, Townsend JRG (2013) High-resolution global maps of 21st-century forest. Science 342: 850-853.

Haslauer E, Delmelle EC, Keul A, Blaschke T, Prinz T (2015) Comparing subjective and objective quality of life criteria: A case study of green space and public transport in Vienna, Austria. Social Indicators Research 124: 911-927.

INEGI (2000) Síntesis de resultados: Zona Metropolitana de Monterrey. XII Censo General de Población y Vivienda 2000. Publicación decenal. Primera edición. Instituto Nacional de Geografía e Informática. Aguascalientes, México. 164p. 
INEGI (2017) Anuario Estadístico y Geográfico de Nuevo León. Instituto Nacional de Geografía e Informática. Aguascalientes, México. 614p.

Jiménez PJ, Cuellar RLG, Treviño GEJ (2013) Áreas Verdes del Municipio de Monterrey. Gobierno Municipal de Monterrey 2012-2015. 21p.

Kweon BS, Marans RW, Yi CW (2016) Parks and quality of life: Differences among african american and white residents. Landscape Journal 35: 97-108.

Manzanilla-Quiñones U, Pozo-Montuy G, Delgado-Valerio P, Martínez-Sifuentes AR, Aguirre-Calderón OA (2021) Escenarios climáticos (CMIP-5) para la Reserva de la Biosfera Pantanos de Centla, Tabasco, México. Ecosistemas y Recursos Agropecuarios Núm. Esp. I: e2588. Doi: 10.19136/era.a8nl.2588.

Marans RW (2015) Quality of urban life and environmental sustainability studies: Future linkage opportunities. Habitat International 45: 47-52.

Molina SA, Delgado P, González-Rodríguez A, González C, Gómez-Tagle RAF, López-Toledo L (2019) Spatiotemporal approach for identification of critical conservation areas: a case study with two pine species from a threatened temperature forest in Mexico. Biodiversity and Conservation 28: 1863-1883.

Navarro-Tec S, Orozco-del Castillo MG, Valdiviezo-Navarro JC, Ordaz-Bencomo DR, Moreno-Sabido MR, BernejoSabbagh C (2018) Análisis del crecimiento urbano y su relación con el incremento de temperaturas en la ciudad de Mérida utilizando imágenes satelitales. Research in Computing Science 147: 285-294.

Pena-Salmon CA, Rojas-Caldelas RI (2009) Methodology for planning urban green areas: the case of Mexicali, Baja California, México. Sustainable Development and Planning 4: 33-41.

Pérez G, Muñoz N (2006) Teledetección: nociones y aplicaciones. Universidad de Salamanca. Salamanca, España. 359p.

Priego G (2011) El valor de los espacios verdes urbanos. Naturaleza y Sociedad. Ministerio de Medio Ambiente y Medio Ambiente Rural y Marino. Jerez de la Frontera, Cádiz, España. 109p.

QGIS (2018) Un Sistema de Información Geográfica libre y de código abierto. Versión 3.2.3. https://qgis.org/es/ site/. Fecha de consulta 15 de febrero de 2020.

R Core Team (2020) R: a language and environment for statistical computing. R Foundation for Statistical Computing. Viena, Austria. Versión 4.0. htpp://www.r-project.org. Fecha de consulta: 15 de mayo de 2020.

Reyes AC (2012) Gestión integral de áreas verdes urbanas: un nuevo campo profesional de la arquitectura de paisaje. En: Mazari HM, Wiener CG (Comps.) Arquitectura de paisaje, obras, proyectos y reflexiones. Primera edición. Universidad Nacional Autónoma de México. México. pp: 161-169.

Rouse JW, Haas RH, Schell JA, Deering DW (1974) Monitoring vegetation systems in the Great Plains with ERTS. In: Freden SC, Mercanti EP, Becker M (ed) Third earth resources technology satellite 1 Symposium. Volume I: Technical Presentations. Washington, D.C., USA. pp: 309-317.

Secretaría de Desarrollo Sustentable del Estado de Nuevo León (2017) Estrategia de desarrollo urano del Estado. https://www.nl.gob.mx/sites/default/files/presentacion_instalacion_cotdunl-final-.pdf. Fecha de consulta: 07 de marzo de 2021.

UN (2019) World urbanization prospects: The 2018 revision (ST/ESA/SER.A/420). New York, USA: United Nations. United Nations, Department of Economic and Social Affairs, Population Division. https://population.un. org/wup/Publications/Files/WUP2018-Report.pdf. Fecha de consulta: 06 de marzo de 2021. 
van den Berg AE, Maas J, Verheij RA, Groenewegen PP (2010) Green space as a buffer between stressful life events and health. Social Science \& Medicine 70: 1203-1210. 